\title{
Quiescence and Subsequent Anterior Chamber Inflammation in Adalimumab-treated Pediatric Noninfectious Uveitis
}

\author{
Sooyeon Choe ${ }^{1}$, Jang Won $\mathrm{Heo}^{2}$, Baek-Lok $\mathrm{Oh}^{1}$ \\ ${ }^{1}$ Department of Ophthalmology, Seoul National University Hospital, Seoul National University College of Medicine, Seoul, Korea \\ ${ }^{2}$ SNU Seoul Eye Clinic, Seoul, Korea
}

Purpose: To evaluate the effect of adalimumab in pediatric uveitis and subsequent changes in anterior chamber inflammation following the inactivation of uveitis.

Methods: In this retrospective study, patients with noninfectious uveitis younger than 18 years of age who were treated with adalimumab for more than 12 months were included. The rate of complete suppression and the relapse in anterior chamber inflammation following the initiation of adalimumab therapy were evaluated using anterior chamber cell score and laser flare photometry (LFP) values, if available. Changes in visual acuity and the sparing effect of topical steroid agents were also evaluated.

Results: Among 22 eyes of 12 pediatric uveitis patients enrolled, 13 eyes were associated with juvenile idiopathic arthritis and nine eyes had idiopathic uveitis. The mean \pm standard deviation age was $10.2 \pm 3.6$ years. Types of uveitis included anterior uveitis $(n=17)$ and panuveitis $(n=5)$. Quiescence was observed in 14 eyes $(63.6 \%)$ at 3 months and in 21 eyes $(95.5 \%)$ at 12 months after initiation, respectively. After achieving inactive uveitis, uveitis relapsed in two eyes at 6 months, even with adalimumab treatment. In 11 eyes, anterior chamber showed $0.5+$ cell scores during the rest of the follow-up period and one of those eyes met the criteria for the relapse based on LFP values. The dosage of topical steroids decreased significantly at 3,9 , and 12 months after the initiation of therapy $(p \leq 0.05)$. Visual acuity did not show improvement. There were no severe adverse effects of anti-tumor necrosis factor- $\alpha$ treatment reported.

Conclusions: In this study, adalimumab achieved a quiescent state in most eyes with pediatric noninfectious uveitis for 12 months with a relapse rate of 9.5\%. LFP values together with the anterior chamber cell score can be utilized to monitor the improvement or relapse in anterior chamber inflammation in pediatric noninfectious uveitis.

Key Words: Adalimumab, Anti-tumor necrosis factor-alpha, Laser flare photometry, Pediatric uveitis, Recurrence

Pediatric uveitis is a rare disease with an estimated yearly incidence of 4.3 per 100,000 in children and a preva-

Received: January 3, 2020 Final revision: February 28, 2020

Accepted: March 23, 2020

Corresponding Author: Baek-Lok Oh, MD. Department of Ophthalmology, Seoul National University Hospital, Seoul National University College of Medicine, 101 Daehak-ro, Jongro-gu, Seoul 03080, Korea. Tel: 822-2072-3740, Fax: 82-2-741-3187, E-mail: baeklok@snu.ac.kr lence of 27.9 per 100,000 [1,2]. Ocular complications including cataract, glaucoma, and macular edema have been reported to affect up to $76 \%$ of all cases [3]. Moreover, there are certain challenges in treating pediatric uveitis patients such as delayed diagnosis, extended burden over time, the risk of amblyopia, difficulty of ocular examinations, and limited treatment options due to complications [2]. 
Conventional treatments for pediatric noninfectious uveitis include topical corticosteroids; peribulbar and intravitreal corticosteroids; systemic corticosteroids; and immunomodulatory agents such as methotrexate (MTX), azathioprine, cyclosporine, and mycophenolate mofetil [4]. Recently, new treatment options encompassing biologic agents, such as anti-tumor necrosis factor (TNF)- $\alpha$ therapy, have emerged $[5,6]$. Adalimumab is a fully human monoclonal antibody that acts against TNF- $\alpha$ and which has been used to treat rheumatoid arthritis, juvenile idiopathic arthritis (JIA), psoriatic arthritis, ankylosing spondylitis, and Crohn's disease. In 2017, SYCAMORE, a well-designed randomized controlled trial, provided evidence for the efficacy of adalimumab in pediatric uveitis-associated JIA [7]. Although this pivotal study reported a reduction in the risk of treatment failure in the patient group treated with adalimumab, the relapse rate after obtaining quiescent uveitis was not evaluated. In practice, data concerning the relapse rate after achieving inactive uveitis with adalimumab and the optimal extended treatment strategy can be helpful in the successful management of pediatric patients with noninfectious uveitis.

Since the establishment of the evaluation system from the Standardization of Uveitis Nomenclature (SUN) working group in 2005, the grading schemes for anterior chamber cells and flare have been the standard evaluation methods in anterior chamber assessment [8]. However, since the anterior chamber cell grading score system is subjective and non-quantitative measurement, it might not be sufficient for evaluating the activity of inflammation in pediatric uveitis $[9,10]$. Moreover, several groups support that the laser flare photometry (LFP) value may be a more useful biomarker of inflammation activity in uveitis and should be adopted to evaluate the improvement and worsening of anterior chamber inflammation in patients with uveitis [1113].

Until now, evidence supporting optimal management methods for pediatric uveitis is still limited, including regarding the response rate of anti-TNF- $\alpha$, the relapse rate of inflammation after the inactivation of uveitis, and the ideal tool to use to assess inflammation activity. Therefore, in this study, we sought to investigate the efficacy of adalimumab treatment in pediatric noninfectious uveitis and the relapse of inflammation, which was determined by conventional anterior chamber cell grade scheme and validated by LFP value.

\section{Materials and Methods}

\section{Ethics statement}

This study was a retrospective cohort study approved by the institutional review board of Seoul National University Hospital (1810-114-982) and which adhered to the tenets of the Declaration of Helsinki. Informed consent was waived because of the retrospective nature of the study and the analysis used anonymous clinical data.

\section{Subjects}

We reviewed the medical records of 22 eyes of 12 pediatric uveitis patients treated with adalimumab for more than 12 months at Seoul National University Children's Hospital between January 2015 and June 2019. Adalimum$a b$ was injected in patients with noninfectious uveitis refractory or intolerant to topical steroid therapy and MTX aged between two and 18 years old (among them, one patient was not using MTX due to side effects). Patients who had used other anti-TNF- $\alpha$ inhibitors before were excluded from our analysis.

\section{Data collection and analysis}

The following demographic data were collected from electronic medical charts: sex, laterality, age at diagnosis, underlying systemic disease (e.g., JIA, idiopathic uveitis), baseline therapy, type of uveitis (e.g., anterior, panuveitis), lens status, and ocular complications of uveitis. Adalimumab was injected subcutaneously every two weeks and the dosage was adjusted individually in relation to the patient's body surface area. (i.e., $20 \mathrm{mg}$ for patients $<30 \mathrm{~kg}$; otherwise, $40 \mathrm{mg}$ ). The underlying systemic disease was diagnosed by a pediatrician.

The primary outcome was the proportion of inactive inflammation (cell grade 0) of the anterior chamber in response to adalimumab treatment and the subsequent relapse rate of anterior chamber inflammation. We assessed outcomes every 3 months from the start of adalimumab injection. The anterior chamber cells were counted in the field size of a $1 \mathrm{~mm} \times 1 \mathrm{~mm}$ slit beam and the severity of the anterior chamber inflammation was graded using the grading system of the SUN working group. Recurrence was defined as a two-step increase in anterior chamber in- 
flammation [8]. Secondary outcomes included LFP values as assessed by the Kowa FM-500 Flare Meter (Kowa, Tokyo, Japan), change in visual acuity, and the frequency of topical corticosteroid usage during the adalimumab regimen. LFP involves the measurement of scattered light from flare or cells in the anterior chamber. Here, a helium-neon or diode laser is used as the incoming light source; then, a photomultiplier detects the scattered light and a computer processes it [14]. The intensity of the scattered light is calculated as the flare count and expressed in photon counts per $\mathrm{ms}(\mathrm{ph} / \mathrm{ms})$. When compared with conventional slit-lamp assessment, a scale from one to four in slit-lamp flare evaluation is comparable to a scale from 4 $\mathrm{ph} / \mathrm{ms}$ (the normal flare value in a noninflamed eye) to values as high or greater than $1,000 \mathrm{ph} / \mathrm{ms}$ in LFP [15]. Due to the limited availability of the machine, nine patients in this study could undergo LFP. Recurrence as indicated by LFP value was defined as an increase of $20 \%$ over the preceding value of LFP and/or an absolute increase of $3 \mathrm{ph} / \mathrm{ms}$ when minimal flare values were below $15 \mathrm{ph} / \mathrm{ms}$ [16].

The outcome values were presented as means \pm standard deviations. Statistical analysis was conducted using Stata ver. 13.0 (Stata Corp., College Station, TX, USA) and R ver. 3.6.1 (http://www.R-project.org; R Foundation for Statistical Computing, Vienna, Austria). A normality test was performed by applying the Shapiro-Wilk test and comparisons between each 3-month interval visits were made with the paired $t$-test and Wilcoxon signed-rank test. Statistical significance was defined as $p \leq 0.05$.

\section{Results}

\section{Patient demographics and clinical and ocular charac- teristics}

A total of 22 eyes of 12 patients were included in this study. Demographic characteristics of the patients $(n=12)$ are shown in Table 1. All patients were Korean by ethnicity. Among them, seven patients $(66.7 \%)$ were diagnosed with JIA and five patients (33.3\%) were idiopathic. Among the JIA patients, six had polyarthritis and one had oligoarthritis. Among idiopathic uveitis cases, four patients did not have any inflammatory signs in the posterior segments, while one patient showed retinal vascular sheathing and diffuse capillary leakage during fluorescein angiography.
Table 1. Demographic characteristics of children with noninfectious pediatric uveitis

\begin{tabular}{lc}
\hline Characteristics & Value \\
\hline No. of children (eyes) & $12(22)$ \\
Sex & \\
Male & $3(25.0)$ \\
Female & $9(75.0)$ \\
Laterality & \\
Unilateral & $2(16.7)$ \\
Bilateral & $10(83.3)$ \\
Age (yr) & $3.6(6.5-17.2)$ \\
Systemic diagnosis & $7(58.3)$ \\
Juvenile idiopathic arthritis & $1(8.3)$ \\
$\quad$ Oligoarticular type & $6(50.0)$ \\
$\quad$ Polyarticular type & $5(41.7)$ \\
Idiopathic uveitis & \\
Baseline therapy & $1^{*}(8.3)$ \\
No systemic medication & $2(16.7)$ \\
Oral steroid + immunosuppressive & \\
therapy & $2.0 \pm 0.7(1.0-3.6)$ \\
Immunosuppressive therapy & \\
Duration of follow-up (yr) &
\end{tabular}

Values are presented as number (\%) or mean \pm standard deviation (range).

* One patient failed to continue systemic steroids or immunosuppressive agents due to side effects including gastrointestinal trouble.

The mean age was $10.2 \pm 3.6$ years (range, 6.5 to 17.2 years) and the mean follow-up duration was $2.0 \pm 0.7$ years (range, 1.0 to 3.6 years).

Ocular characteristics of the eyes $(n=22)$ are summarized in Table 2. The proportions of anterior uveitis and panuveitis were $77.3 \%$ and $22.7 \%$, respectively. The mean baseline visual acuity was 0.53 logarithm of the minimum angle of resolution (range, -0.08 to 1.85 ).

\section{Primary outcome}

The proportion of the quiescent anterior chamber increased gradually according to time in response to adalimumab therapy as shown in Fig. 1. Inactive uveitis was observed in 14 (63.6\%), 19 (86.4\%), 20 (90.9\%), and 21 (95.5\%) eyes at 3, 6, 9, and 12 months of follow-up, respectively. In two eyes of two patients, anterior chamber relapses with 
Table 2. Baseline ocular characteristics of eyes with pediatric uveitis

\begin{tabular}{lc}
\hline Characteristics & Value \\
\hline No. of eyes & 22 \\
Uveitis type & \\
Anterior uveitis & $17(77.3)$ \\
Panuveitis & $5(22.7)$ \\
Lens status & \\
Clear & $3(13.6)$ \\
Cataract & $11(50.0)$ \\
Pseudophakia & $8(36.4)$ \\
Anterior chamber cell score & \\
1+ & $16(72.7)$ \\
$2+$ & $5(22.7)$ \\
$3+$ & $1(4.5)$ \\
$4+$ & $0(0.0)$ \\
Baseline topical therapy & \\
Yes & $20(90.9)$ \\
No & $2(9.1)$ \\
Ocular complications & $0.53(0.0)$ \\
Band keratopathy & $0.35(0.014$ to 1.2$)$ \\
Posterior synechiae & $136.1(4.5$ to 436$)$ \\
Optic disc swelling & \\
Neovascularization & \\
\hline
\end{tabular}

Values are presented as number (\%) or mean \pm standard deviation (range) unless otherwise indicated.

$\mathrm{VA}=$ visual acuity; $\log \mathrm{MAR}=$ logarithm of the minimum angle of resolution; LFP = laser flare photometry.

${ }^{*}$ Seventeen eyes were measured at baseline among 22 eyes.

cell scores of $2+$ were observed at 3 months after achieving quiescence of the anterior chamber.

\section{Secondary outcomes}

In this study, the anterior chamber flare was quantified by LFP. Seventeen eyes of nine patients were measured by LFP at baseline and a total of eight eyes of four patients were analyzed by LFP throughout the study period without any missing data. The proportions of participants whose LFP values improved by more than $30 \%$ were

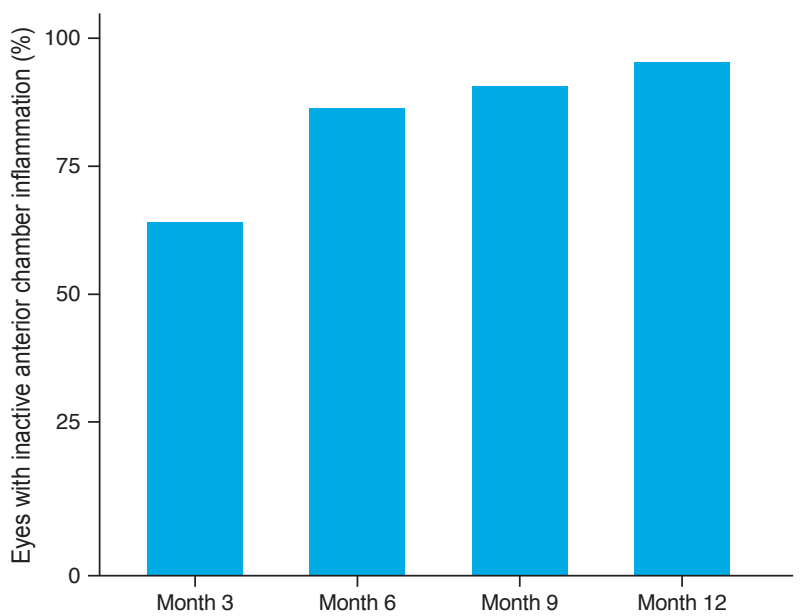

Fig. 1. Cumulative proportion of eyes with quiescent anterior chamber inflammation (cell grade 0 ) after adalimumab treatment in noninfectious pediatric uveitis.

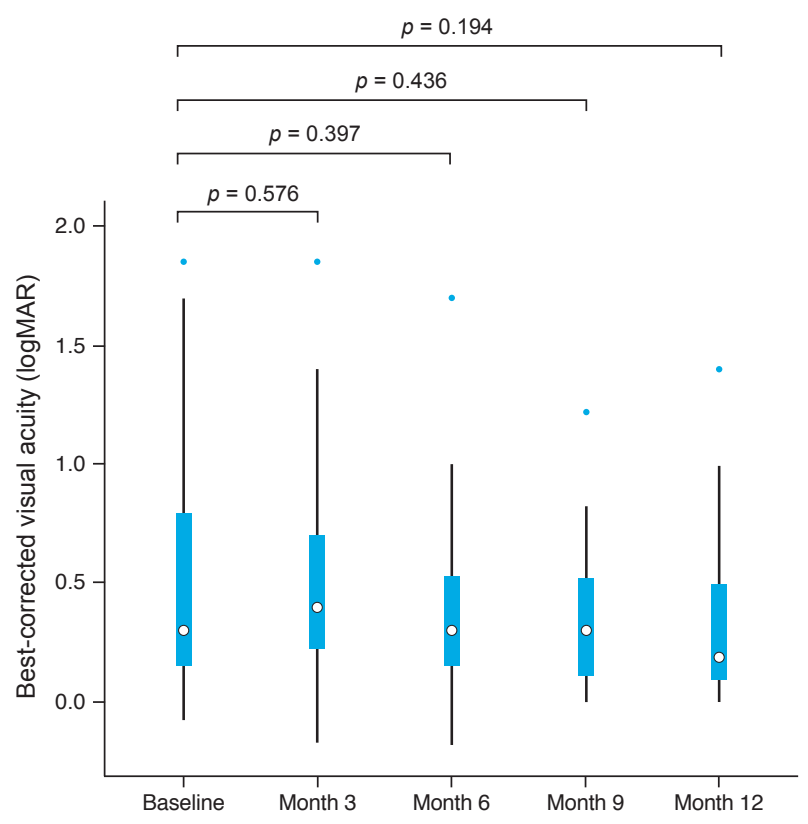

Fig. 2. Best-corrected visual acuities after adalimumab treatment in noninfectious pediatric uveitis (Wilcoxon signed-rank test). $\log \mathrm{MAR}=\operatorname{logarithm}$ of the minimum angle of resolution.

$62.5 \%, 75 \%, 100 \%$, and $100 \%$ at $3,6,9$, and 12 months of treatment, respectively. After the inactivation of uveitis, $0.5+$ cells in the anterior chamber developed in 11 eyes during the rest of the follow-up period; one eye met the criteria of relapse based on LFP results.

Changes in best-corrected visual acuity are depicted in Fig. 2. Visual acuities did not vary significantly $(p>0.05$, 


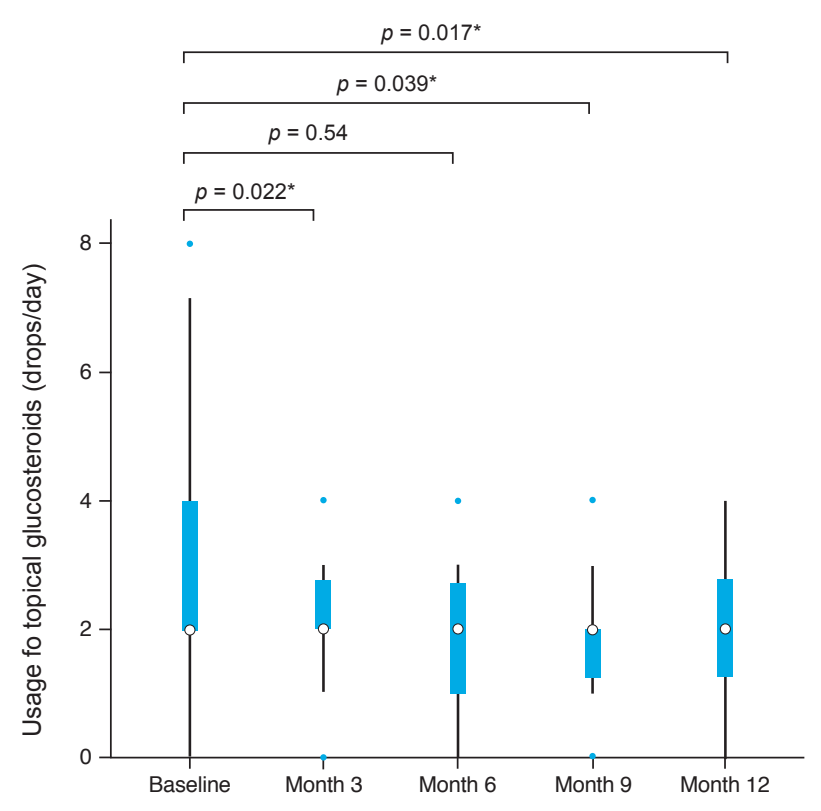

Fig. 3. Usage of topical corticosteroid after adalimumab treatment in noninfectious pediatric uveitis (paired $t$-test). ${ }^{*} p \leq 0.05$.

Wilcoxon signed-rank test). Adalimumab therapy significantly reduced the usage of topical steroid agents at 3,9 , and 12 months after adalimumab treatment $(p \leq 0.05$, paired $t$-test) (Fig. 3). No patient experienced serious adverse effects of adalimumab therapy such as tuberculosis, hepatitis, malignancy, hematologic problems, or serious infections during the follow-up period. Furthermore, only one patient reported the side effect of recurrent molluscum contagiosum.

\section{Discussion}

In this retrospective study, the use of adalimumab was advantageous in achieving quiescence of the anterior chamber inflammation in refractory pediatric uveitis for 12 months of follow-up without severe adverse effects, with a relapse rate of $9.5 \%$.

Anterior chamber inflammation became inactive in 21 of 22 eyes (95.5\%) after one year of treatment with adalimumab. When assessing the anterior chamber inflammation by LFP value change, a similar trend was observed. The decreasing LFP values throughout the adalimumab medication period were consistent with those from a previous study reported by Quartier et al. [12].

We reported that adalimumab reduces topical steroid agent dosage significantly (except for considering the topical steroid agent-sparing effect at 6 months after the initiation of adalimumab therapy). This result is in line with previous studies. Deitch et al. [17] in a study of 43 eyes of 24 patients reported that $82.4 \%$ of eyes discontinued using topical steroid eye drops after adalimumab therapy. Castiblanco et al. [18] found that $77 \%$ of patients (13/17 eyes) treated with adalimumab achieved steroid-free remission. Ardoin et al. [19] reported that $69 \%$ of eyes were free from topical steroid use at the end of the first year of follow-up after adalimumab treatment.

Our study revealed a recurrence rate of 9.5\% (2 / 21 eyes). Two eyes showed increased anterior chamber cell grading to $2+$ at 6 months from the start of adalimumab therapy. Sen et al. [20] documented that six of 19 eyes showed recurrence during 12 months with the use of adalimumab. Of these six eyes, five relapsed between 3 and 12 months of therapy, but the study did not collect anterior chamber cell data between 3 and 12 months of therapy. Deitch et al. [17] reported that $33.3 \%$ of 43 eyes relapsed at 6 months and $4.5 \%$ did so at 9 months. Although the recurrence rate differs between studies, adalimumab showed an initial effect with relapse between 3 and 12 months after the start of treatment. In the present study, 11 eyes showed increased cell grade from 0 to $0.5+$ but did not meet the criteria of recurrence defined by SUN. At this point, we analyzed LFP values to see whether anterior chamber inflammation did not satisfy the requirement for "recurrence" according to LFP values among patients who have undergone LFP. Among 11 eyes with increment of anterior chamber cell grade from 0 to $0.5+, 10$ eyes underwent LFP and only one of these 10 eyes met the criteria for "recurrence" according to LFP value. Our result supports the SUN definition that the increment of anterior chamber cell grade from 0 to $0.5+$ should not be regarded as a relapse of uveitis.

Anti-TNF- $\alpha$ therapy potentially can complicate many serious adverse events including infection, malignancy, other hematologic disorders, demyelinating disorders reported in patients with rheumatic disease [21]. One study reported that reported no adverse events occurred in uveitis patients during 18 months of follow-up [12], while another found higher incidence rates of adverse events during the same length of follow-up, including $13 \%$ for serious adverse events and $8 \%$ for infection or infestation events [7]. Among 12 patients in this study, there were no 
serious adverse events seen throughout 12 months of adalimumab medication. Only one patient experienced recurrent molluscum contagiosum on his skin.

Limitations of this study include its retrospective design and small patient cohort. Further, not all patients were evaluated by LFP at each visit. Future prospective studies with systematic acquisition of LFP values can help understand the role of LFP in monitoring the improvement and worsening of anterior chamber inflammation in pediatric uveitis.

In conclusion, adalimumab could be effective and safe for noninfectious pediatric uveitis. LFP values may suggest both improvement and worsening of anterior chamber inflammation in pediatric noninfectious uveitis patients.

\section{Conflict of Interest}

No potential conflict of interest relevant to this article was reported.

\section{References}

1. Paivonsalo-Hietanen T, Tuominen J, Saari KM. Uveitis in children: population-based study in Finland. Acta Ophthalmol Scand 2000;78:84-8.

2. Zierhut M, Michels H, Stubiger N, et al. Uveitis in children. Int Ophthalmol Clin 2005;45:135-56.

3. Cann M, Ramanan AV, Crawford A, et al. Outcomes of non-infectious Paediatric uveitis in the era of biologic therapy. Pediatr Rheumatol Online J 2018;16:51.

4. Smith JA, Mackensen F, Sen HN, et al. Epidemiology and course of disease in childhood uveitis. Ophthalmology 2009;116:1544-51.

5. Reiff A, Takei S, Sadeghi S, et al. Etanercept therapy in children with treatment-resistant uveitis. Arthritis Rheum 2001;44:1411-5.

6. Carrasco R, Smith JA, Lovell D. Biologic agents for the treatment of juvenile rheumatoid arthritis: current status. Paediatr Drugs 2004;6:137-46.

7. Ramanan AV, Dick AD, Jones AP, et al. Adalimumab plus methotrexate for uveitis in juvenile idiopathic arthritis. $N$ Engl J Med 2017;376:1637-46.

8. Jabs DA, Nussenblatt RB, Rosenbaum JT; Standardization of Uveitis Nomenclature (SUN) Working Group. Standard- ization of uveitis nomenclature for reporting clinical data. Results of the First International Workshop. Am J Ophthalmol 2005;140:509-16.

9. Herbort CP, Tugal-Tutkun I. The importance of quantitative measurement methods for uveitis: laser flare photometry endorsed in Europe while neglected in Japan where the technology measuring quantitatively intraocular inflammation was developed. Int Ophthalmol 2017;37:469-73.

10. Heiligenhaus A, Foeldvari I, Edelsten C, et al. Proposed outcome measures for prospective clinical trials in juvenile idiopathic arthritis-associated uveitis: a consensus effort from the multinational interdisciplinary working group for uveitis in childhood. Arthritis Care Res (Hoboken) 2012;64:1365-72.

11. Bernasconi O, Papadia M, Herbort CP. Sensitivity of laser flare photometry compared to slit-lamp cell evaluation in monitoring anterior chamber inflammation in uveitis. Int Ophthalmol 2010;30:495-500.

12. Quartier P, Baptiste A, Despert V, et al. ADJUVITE: a double-blind, randomised, placebo-controlled trial of adalimumab in early onset, chronic, juvenile idiopathic arthritis-associated anterior uveitis. Ann Rheum Dis 2018;77:100311.

13. Herbort CP, Guex-Crosier Y, de Ancos E, Pittet N. Use of laser flare photometry to assess and monitor inflammation in uveitis. Ophthalmology 1997;104:64-71.

14. Sawa M, Tsurimaki Y, Tsuru T, Shimizu H. New quantitative method to determine protein concentration and cell number in aqueous in vivo. Jpn J Ophthalmol 1988;32:13242.

15. el-Maghraby A, Marzouki A, Matheen TM, et al. Reproducibility and validity of laser flare/cell meter measurements as an objective method of assessing intraocular inflammation. Arch Ophthalmol 1992;110:960-2.

16. Tugal-Tutkun I, Herbort CP. Laser flare photometry: a noninvasive, objective, and quantitative method to measure intraocular inflammation. Int Ophthalmol 2010;30:453-64.

17. Deitch I, Amer R, Tomkins-Netzer O, et al. The effect of anti-tumor necrosis factor alpha agents on the outcome in pediatric uveitis of diverse etiologies. Graefes Arch Clin Exp Ophthalmol 2018;256:801-8.

18. Castiblanco C, Meese H, Foster CS. Treatment of pediatric uveitis with adalimumab: the MERSI experience. $J$ AAPOS 2016;20:145-7.

19. Ardoin SP, Kredich D, Rabinovich E, et al. Infliximab to treat chronic noninfectious uveitis in children: retrospec- 
tive case series with long-term follow-up. Am J Ophthalmol 2007;144:844-9.

20. Sen ES, Sharma S, Hinchcliffe A, et al. Use of adalimumab in refractory non-infectious childhood chronic uveitis: effi- cacy in ocular disease: a case cohort interventional study. Rheumatology (Oxford) 2012;51:2199-203.

21. Antoni C, Braun J. Side effects of anti-TNF therapy: current knowledge. Clin Exp Rheumatol 2002;20:S152-7. 